\title{
Synchronous frequency grid dynamics in the presence of a large-scale population of photovoltaic panels
}

\author{
Andrea Peruffo, Alessandro Abate \\ Department of Computer Science \\ University of Oxford \\ Oxford, UK \\ \{name.surname\}@cs.ox.ac.uk
}

\author{
Emeline Guiu, Patrick Panciatici \\ Research and Development \\ RTE France \\ Paris, France \\ \{name.surname\}@rte-france.com
}

\begin{abstract}
The increased relevance of renewable energy sources has modified the behaviour of the electrical grid. Some renewable energy sources affect the network in a very distributed manner: whilst each unit has little influence, a large population can have a significant impact on the global network, particularly in the case of synchronised behaviour. This work investigates the behaviour of a large, heterogeneous population of photovoltaic panels connected to the grid. We employ Markov models to represent the aggregated behaviour of the population, while the rest of the network (and its associated consumption) is modelled as a single equivalent generator, accounting for both inertia and frequency regulation. Analysis and simulations of the model show that it is a realistic abstraction, and quantitatively indicate that heterogeneity is necessary to enable the overall network to function in safe conditions and to avoid load shedding.
\end{abstract}

Index Terms-photovoltaic panels, heterogeneity, frequency, population models, Markov models

\section{INTRODUCTION}

Over the last few years the penetration of renewable energy considerably increased all over the world [1], [2], [3]. As a consequence, their contribution to the electric grid significantly gained importance both for power generation and network reliability. Advances of this technology, decreases in price, and subsidies have been prompting their installation and sustaining their use. In many European countries solar panels have prioritised connection onto the network over other energy generation sources [4].

Wind, solar and geothermal power are also broadly referred to as "distributed generation", meaning that the corresponding generation devices are dispersed over a large area. This is in contrast with conventional power generation systems, and results in a variety of small power-generating systems rather than single machines.

Further heterogeneity is due to their extended employment: over years of use the performance of such devices naturally degrades, manufacturers modify production materials and standards for new devices, and countries change regulations and requirements. Up to a few years ago, distributed energy sources were subjected to connection requirements respecting the operational principles of the distribution network, which was modelled around passive loads and a small number of large power generation units. This led to a number of electrical incidents in areas with a significant amount of distributed generation, which could not be handled with those regulatory designs [5]. With newer regulations [6] instead, every power generation unit is subject to the same requirements: as such, both conventional and renewable sources must be operative in a larger interval of network frequency. New requirements do not hold over household solar devices have been installed for decades, many of which have been manufactured following previous regulations.

In this work we present a modelling framework for large populations of photovoltaic (PV) panels connected to the grid. Each panel is characterised with a frequency sensor and an internal clock. It samples the local grid frequency to ensure that it lies inside a predefined interval of allowed values, and it clocks the amount of time the frequency remains inside the given interval. Two components are key to model the behaviour of a panel connected to the grid: the working interval of grid frequency, and the time delay necessary to perform a safe connection to the network. In [7] we built two models to describe the aggregation of a large population of photovoltaic panels connected to the grid. Both are built as discrete time Markov chains, and are shown to be good representation of the system of interest, and to be prone to analysis and scalable simulations. In this paper we address the issue of evaluating the relationship between the allowed working interval and the stability of the electrical network. The aim of this work is assessing the risk of load shedding with a growing photovoltaic population: we focus especially on the disconnection and reconnection thresholds of solar devices. Simulations display the validity of our approach, and further provide evidence for the need of implementation of a larger working interval of frequencies for PV panels connected to the grid.

In the remainder of the paper, we describe the behaviour in frequency of a PV panel (Section II). Thereafter in Section III we describe two Markov chain models for the aggregation of a large population of devices, encompassing their intrinsic 
heterogeneity. Section IV introduces a grid model for Continental Europe, based on ENTSO-E work [8]. In Section V we present several case studies to understand connections between population heterogeneity and stability of the network. Finally, the conclusions of this paper are drawn in Section VI.

\section{Model of A Single PV PANEL}

A physical description of photovoltaic panels connected to the electric network may be found in literature, see e.g. [9]. We report it here for clarity and completeness. We consider mainly household devices, i.e. installed on the roof of a common house. This sort of population is more prone to heterogeneity, due to different manufacturers, ages, weather conditions, regulations. A panel is connected to the electrical grid and samples it at a fixed sampling time. The panel can be either ON or OFF; the switching among these two states depends on two quantities: the network frequency $f(\cdot)$ and a safety time delay $\tau_{r}$. Table I resumes the behaviour of a PV panel considering the combination of the condition on the frequency signal and the time delay. Regulations impose the panel to work, i.e. being in the ON state, when the grid frequency $f$ belongs to the interval $\mathcal{I}_{f}$, a neighbourhood of the nominal frequency $f_{0}=50 \mathrm{~Hz}$. If the frequency exits $\mathcal{I}_{f}$, the panel must disconnect, i.e. be in the OFF state.

We assume the ON-to-OFF transition to be instantaneous, while this assumption does not hold for the symmetric transition OFF-to-ON. The ON-to-OFF transition should in fact be taken as soon as the network in considered not stable. In order to guarantee the safety of the device, a solar panel only connects to a supposed stable network. The network is considered to be stable if the frequency lies within $\mathcal{I}_{f}$ for a predefined amount of time, $\tau_{r}$. The panel is equipped with an internal counter $\tau(\cdot)$ that is increased with time. It is then compared to the time threshold $\tau_{r}$, so that when $\tau(\cdot) \geq \tau_{r}$ the panel will turn ON. However, whenever the frequency signal exits the interval $\mathcal{I}_{f}$ the counter is reset and the panel must wait again $\tau_{r}$ time instants to turn ON. A photovoltaic panel senses the frequency thanks to a digital sensor, so it is reasonable to assume $\tau_{r}$ is a quantity defined in number of samples. For this reason $\tau(\cdot)$ can be considered as a counter, and as such will be modelled in the present work. In many practical applications, sampling time is in the order of $200 \mathrm{~ms}$ while $\tau_{r}$ results around $20 \mathrm{~s}$.

Note that $\mathcal{I}_{f}$ and $\tau_{r}$ are not homogeneous across a population of panels. Our network setting - a continental grid or part of it - comprehends various norms along several countries. Even in the same country regulations may differ year by year leading to inhomogeneity in the frequency interval $\mathcal{I}_{f}$ and in the time delay $\tau_{r}$. Furthermore, digital system are sensitive to noise in the measurements and suffer from ageing of its components: different panels sample the same frequency signal but they may obtain different values for it.

A single PV panel has a negligible effect when connected to the grid. However as the size of populations of these devices is growing, the total power injected on the grid may have important effects on the network frequency dynamics. This in
TABLE I

SWITCHING BEHAVIOUR OF A SINGLE PHOTOVOLTAIC PANEL WITHIN THE POWER NETWORK AT TIME $k$.

\begin{tabular}{|c|c|c|c|}
\hline State $q(k)$ & Frequency measurement & Delay & State $q(k+1)$ \\
\hline OFF & $f(k) \in \mathcal{I}_{f}$ & $\tau(k) \geq \tau_{r}$ & ON \\
\hline ON & $f(k) \in \mathcal{I}_{f}$ & - & ON \\
\hline ON & $f(k) \notin \mathcal{I}_{f}$ & - & OFF \\
\hline OFF & $f(k) \in \mathcal{I}_{f}$ & $\tau(k)<\tau_{r}$ & OFF \\
\hline
\end{tabular}

turn directly influences the behaviour of single panels, in a feedback fashion. The aim of this work is to develop a model for a large population of photovoltaic panels, and perform simulation to show its predictive value. The model needs to address the underlying heterogeneity arisen from different manufacturers, makes, age, regulations.

Another challenging feature of real power systems is the unpredictability of the power output: solar irradiance is the source of the photoelectric effect but weather conditions are often difficult to forecast. In this work we focus on a constant power production. This is a reasonable assumption over small time scales (seconds) and during a clear sunny day. However, this assumption might not hold during a cloudy day: as observed in [10], individual solar plants can significantly vary their outputs over the course of seconds. Weather should be encompassed in the model to have a better description a real system: we plan to add weather behaviour to our model as a stochastic process, as in [11], at a later stage.

\section{Model of A Population of PV PANElS}

In Section II we have considered the behaviour of a single panel, depending on its sampling of the frequency signal. This consideration suggests a discrete time framework as a natural approach to modelling. Further, in order to handle the stochastic nature of the system we utilise a Markov chain model. This enables to analyse the behaviour of the population of panels in a reliable and effective manner. In this section we briefly describe the model a large population of photovoltaic panels - for more details, please refer to [7].

\section{A Markov model without delays}

We assume that disconnection and reconnection thresholds are different for each panel, meaning that a panel connects or disconnects at different frequency values than others. Given a large population of PV panels, we consider the thresholds continuously distributed according to a predefined probability distribution function, that arises from the interpolation of data gathered across Europe.

We now introduce a shift in perspective: from the single $\mathrm{PV}$ panel with a deterministic transition from ON to OFF (and viceversa) we move towards a population of PVPs where panels have a probability to transition from $\mathrm{ON}$ to $\mathrm{OFF}$ (and viceversa). The switching probability at time $k$ is obtained integrating the probability distribution functions: we introduce 


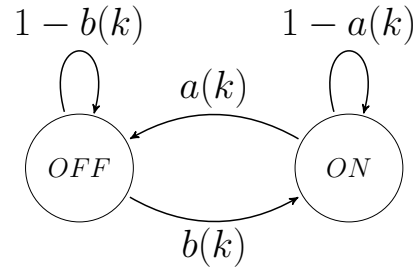

Figure 1. A time inhomogeneous Markov chain model for the aggregated dynamics, without delays.

$a(\cdot)$ as the disconnection probability, and $b(\cdot)$ as the reconnection probability. More formally,

$$
a(k)=\int_{-\infty}^{f(k)} p d f^{d}(u) d u, \quad b(k)=\int_{f(k)}^{\infty} p d f^{r}(u) d u,
$$

where $p d f^{d}$ is the probability density function of a random variable modelling the transition from $\mathrm{ON}$ to $\mathrm{OFF}$, and conversely for $p d f^{r}$. Note that the value of frequency $f(k)$ is one of the two extrema of the integral.

Our model allows to use a two-state Markov chain: $a(\cdot)$ is the ON-to-OFF probability and $(1-a(\cdot))$ is the ON-to-ON one - similarly for $b(\cdot)$ and state OFF. We introduce $x(k)$ as the probability of being in the ON state at time $k$ and $(1-x(k))$ as the probability of being in the OFF state. This allows to write the update equation as

$$
x(k+1)=(1-a(k)) x(k)+b(k)(1-x(k)) .
$$

The expression in Equation (1) shows the time-update of the probability of being ON. Figure 1 shows the Markov chain under consideration.

\section{A Markov model with delays}

We now introduce delays in the model: these are fundamental components in the real behaviour of PV panels. We assume that each panel has an internal counter for the OFF-to-ON transition, which allows the switch after a given delay. We consider to sample the delays from a predefined probability distribution function that provides us with the probability transition values $\tau_{i}$. The value $\tau_{i}$ represents the probability of switching to state $\mathrm{ON}$ given that the panel is waiting for $i$ time instants. In other words, it characterises the probability of transition to state $\mathrm{ON}$ when the frequency lies inside the working threshold for $i$ time instants. We expand the model in the previous section with $n$ states (cf. Fig. 2), each representing the $i$-th waiting instant, defined as $W_{i}, i=1, \ldots, n$. The value $n$ can be chosen as the maximum delay expected for a panel to perform the OFF-to-ON transition.

In each one of the $n$ delay states there are three outgoing transitions: one towards the ON state, one towards state $i+1$, and one back to the OFF state. The probability associated with the third transition is $1-b(k)$, which is the probability of sensing the network frequency outside of the working interval. The first outgoing transition has probability $\tau_{i} b(k)$ : $\tau_{i}$ is the probability to have a time delay that permits the panel to go from state $W_{i}$ to state $\mathrm{ON}$, which can happen

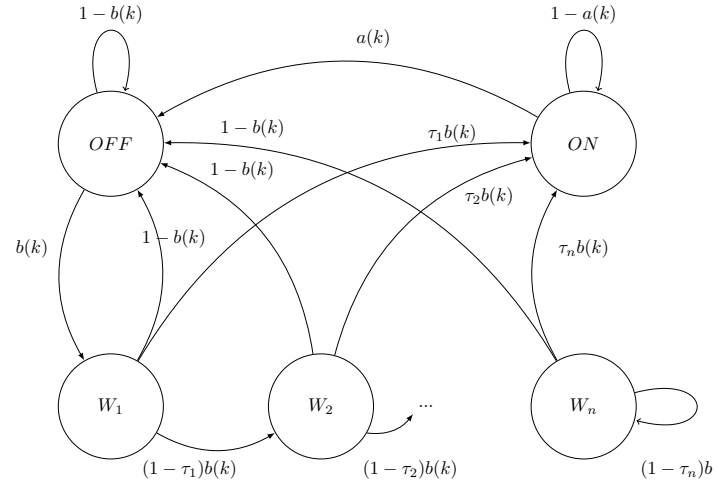

Figure 2. A Markov model for the aggregated dynamics, with delays.

only if the frequency is within the working interval (hence the multiplication by $b(k))$. We assume that $\forall i, \tau_{i} \geq \tau_{i+1}$ and that $\sum_{i} \tau_{i}=1$, so the terms resemble a geometric distribution describing an arrival process or a waiting-time random variable.

Notice that we have tacitly assumed that the probability distributions of the frequency thresholds and time delay are independent. There can be, in reality, some correlation between these two quantities, in which case we need to compute integrals of joint probability distributions - for simplicity in this paper we assumed the uncorrelated case. The dynamics of the Markov chain in Fig. 2 can be summarised as

$\left\{\begin{array}{l}x(k+1)=(1-a(k)) x(k)+b(k) \sum_{i=1}^{n} \tau_{i} w_{i}(k) \\ w_{1}(k+1)=b(k)\left[1-x(k)-\sum_{i=1}^{n} w_{i}(k)\right] \\ w_{i}(k+1)=b(k)\left(1-\tau_{i-1}\right) w_{i-1}(k) \\ w_{n}(k+1)=b(k)\left[\left(1-\tau_{n-1}\right) w_{n-1}(k)+\left(1-\tau_{n}\right) w_{n}(k)\right]\end{array}\right.$

where $x(k)$ and $w_{i}(k)$ represent the probability of being in the ON state and in state $W_{i}$ respectively, at time $k$. We consider the quantity $x(\cdot)$ as the portion of panels $\mathrm{ON}$; similarly, $w_{i}(k)$ is the portion of panels waiting to turn ON for $i$ time steps; the quantities $a(k), b(k)$ are the integrals of the distributions of frequency thresholds in the population of panels.

Let us stress an important detail of the model in Equation (2). Tests on real devices [12], [13] have evidenced an interval of time in which no panel switches on; after this interval, panels switch on according to a roughly geometric probability distribution. The latter probabilistic occurrences are characterised with the $W_{i}$ states. On the other hand, in order to model the first time interval - around $20 s$ - we can include new "pure-waiting" states (their number depending on the minimum delay PV panels switch on). Formally, these states have $\tau_{i}=0$, namely zero probability to transition to the ON state. We may add these new states - and corresponding dynamical equations - to the model, without invalidating its analysis. However for simplicity and brevity we will continue the formal analysis of the model without them, and directly insert them in the case studies. 


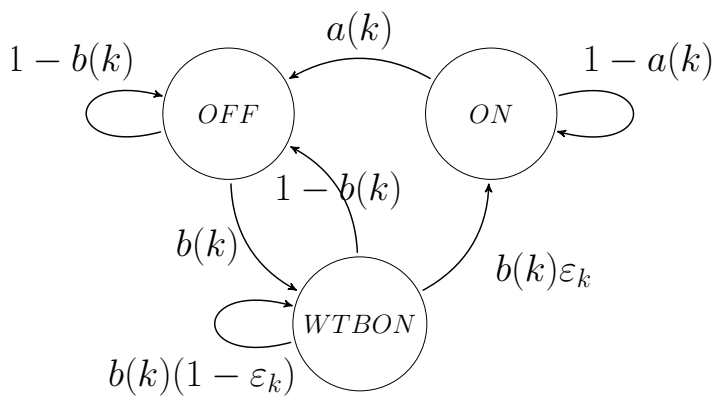

Figure 3. Abstraction of the Markov model in Fig. 2, with dynamics of aggregated delays.

\section{An abstraction of the Markov model with delays}

The model described in the previous section is complete, descriptive and reliable [7], however can present a large number of states, namely $(n+2): n$ waiting states, and states $\mathrm{ON}$ and OFF. In order to overcome potential computational overheads, we propose a further abstraction: we lump the $n$ waiting states into a single state that encapsulates the entire portion of the PV panels waiting to turn on. We express this lumping with a new variable $y$ obtained as the sum of the probabilities $w_{i}(\cdot)$, i.e. $y(\cdot)=\sum_{i} w_{i}(\cdot)$.

Equation (2) can be accordingly modified aggregating the corresponding dynamics, which leads to the simplified system

$$
\left\{\begin{array}{l}
x(k+1)=(1-a(k)) x(k)+b(k) \varepsilon_{k} y(k) \\
y(k+1)=b(k)(1-x(k))-b(k) \varepsilon_{k} y(k),
\end{array}\right.
$$

where we have introduced a time-varying term $\varepsilon_{k}$ such that

$$
\sum_{i=1}^{n} \tau_{i} w_{i}(k)=\varepsilon_{k} \sum_{i=1}^{n} w_{i}(k), \quad \text { thus } \quad \varepsilon_{k}=\frac{\sum_{i=1}^{n} \tau_{i} w_{i}(k)}{\sum_{i=1}^{n} w_{i}(k)} .
$$

This simplification leads to a smaller three-state Markov chain, depicted in Figure 3, where the transition probabilities are obtained by the multiplication of the terms $a(\cdot)$ and $b(\cdot)$ by the new quantity $\varepsilon_{k}$. The new model is smaller, easier to analyse, and arguably less expensive to simulate. Interestingly, if we assume to know the $(n+2)$-state model but are unable to measure the term $\varepsilon_{k}$, we are able to estimate $\varepsilon_{k}$ just from network frequency measurements. This fact, and more generally the analysis of the stability properties of the model can be found in [7].

\section{Model of The Network Dynamics}

The feedback interconnection of the overall system comprises both the population of PV panels and the underlying network frequency dynamics.

In the previous sections we built a model for the population of solar panels and discussed how the grid frequency influences its dynamics. We now focus on how the population of panels affects the frequency dynamics. It is usual in the power systems to describe the network frequency dynamics via the rotating mass equation in continuous time. However the proposed model naturally unfolds in a discrete time framework: we will show that this modelling choice is also reliable and provides accurate empirical results.

We obtain a continuous-time model of the electric network from the indications contained in the ENTSO-E report [8]. The model includes also the primary control of the grid (designed as an internal feedback loop), in order to simulate a realistic approximation of the network dynamics, where the contribution of PV panels is added. The self-regulation of the load is assumed to be $1 \% / \mathrm{Hz}$, the average network power frequency characteristic of primary control $15000 \mathrm{MW} / \mathrm{Hz}$, and the system start time 10 seconds. ${ }^{1}$

We obtain a second-order transfer function, relating the photovoltaic power (as input) to the frequency change (as output). We discretise the model via the step response invariance method, which guarantees perfect matching of the continuoustime signal with the discrete-time signal at sampling times. We then obtain a second-order discrete transfer function that can be written as

$$
\Delta f(k+1)=\frac{c_{1} z+c_{2}}{z^{2}+d_{1} z+d_{2}} P_{P V}(k),
$$

where $\Delta f(k)=f(k)-f_{0}, f_{0}$ represents the nominal value of the network frequency, $f(k)$ is the value of the frequency at time $k$, while $P_{P V}(k)$ is the power output of the population of PV panels at time $k$. We set

$$
P_{P V}(k)=\bar{p} N x(k),
$$

where $\bar{p}$ is assumed to be the constant power output of a single PV panel, and $N$ is the total number of panels. Note that we utilise a direct proportional relation between power output and portion of panels on. However, considering it to be homogeneous across the whole population might be restrictive. This unique value simplifies the formal analysis on the feedback systems presented in Equations (5) and (6). In the future we plan to integrate topological data on solar irradiance over the region of interest.

Embedding the frequency description in Equation (4) within the dynamics of the Markov chain with $n$ waiting states (2) results in

$$
\left\{\begin{array}{c}
f(k+1)=\alpha_{1} f(k)+\alpha_{2} f(k-1)+ \\
\quad+\beta_{1} x(k)+\beta_{2} x(k-1) \\
x(k+1)=(1-a(k)) x(k)+b(k) \sum_{i=1}^{n} \tau_{i} w_{i}(k) \\
w_{1}(k+1)=b(k)\left[1-x(k)-\sum_{i=1}^{n} w_{i}(k)\right] \\
w_{i}(k+1)=b(k)\left(1-\tau_{i-1}\right) w_{i-1}(k) \\
w_{n}(k+1)=b(k)\left[\left(1-\tau_{n-1}\right) w_{n-1}(k)+\right. \\
\left.\quad+\left(1-\tau_{n}\right) w_{n}(k)\right],
\end{array}\right.
$$

and into the Markov chain with three states (3) to leads to

$$
\left\{\begin{aligned}
f(k+1)= & \alpha_{1} f(k)+\alpha_{2} f(k-1)+ \\
& +\beta_{1} x(k)+\beta_{2} x(k-1) \\
x(k+1)= & (1-a(k)) x(k)+b(k) \varepsilon_{k} y(k) \\
y(k+1)= & b(k)\left(1-x(k)-\varepsilon_{k} y(k)\right) .
\end{aligned}\right.
$$

\footnotetext{
${ }^{1}$ The self regulation of the load is the sensitivity of consumers demand to variations in the system frequency (e.g., a decrease of the frequency results in a decrease of the load), and is generally expressed as $\% / \mathrm{Hz}$ [14]. The system start time is the time that a device needs to accelerate from zero to the working speed.
} 
The models described by Equations (5) and (6) are utilised in the following Case Studies. They will be compared against a realistic model, where panels are described explicitly, to test their effectiveness.

\section{Simulation and Testing Via Case Studies}

In this section we employ the developed aggregated models for the population of PV panels in several scenarios. Previously [7], the quality of the aggregated models has been tested on a simulation benchmark, which has shown their precision.

The present objective is to study the consequences of a "normal incident" (as defined shortly) under varying levels of penetration of solar energy in the network, and under different assumptions on the distribution of the frequency thresholds over the population. In order to simulate this setup, we inject a negative (or positive) step in the frequency loop corresponding to the selected incident.

The Operation Security Network Code [15] divides incidents into three categories: normal, exceptional, and out-ofrange. After a normal incidents the function of the system must be maintained without violations of technical limits. Normal incidents are classified as a loss of up to $2 \mathrm{GW}$ of load, and as a loss of up to $3 \mathrm{GW}$ of power generation. In our simulations we utilise the maximum value defining these incidents, namely a loss of load of $2 \mathrm{GW}$ and a loss of power generation of $3 \mathrm{GW}$, and test the response of the network under different circumstances, as described next. Notice that the loss of load experiments are performed assuming the initial network frequency to be $50.1 \mathrm{~Hz}$ instead of the canonical 50 Hz. In [5], the authors have proved that there is low risk of load shedding in case of a loss of load when $f_{0}=50 \mathrm{~Hz}$. Our simulations (not reported here) confirm their initial findings: in many scenarios, the $2 \mathrm{GW}$ load loss is not sufficient to reach the $50.2 \mathrm{~Hz}$ threshold, and no panels disconnect. When instead $50.2 \mathrm{~Hz}$ is reached, few panels disconnect, the grid frequency decreases and it stabilises. The load-shedding risk with $f_{0}=50 \mathrm{~Hz}$ and in case of a load loss incident is minimum. We simulate two working points for the network: a low consumption scenario $(220 \mathrm{GW})$ and a high consumption scenario $(440 \mathrm{GW})$, in accordance with [5]. In both cases we consider three degrees of penetration of solar energy production in the network, namely $10 \%, 20 \%, 40 \%$.

As a reference, a realistic model is implemented, where the $N$ panels in the population are individually modelled, with their corresponding frequency thresholds and time delay. This explicit model is employed as "ground truth" and compared against the two abstract models, the $(n+2)$-state and the threestate model, presented in the previous sections.

Different regulations on solar inverters behaviour exist for a network such as that in continental Europe. As an example, many countries [5] allow solar inverters to connect and disconnect at ranges of $\pm 200 \mathrm{mHz}$ around the nominal frequency, while others around $\pm 600 \mathrm{mHz}$. In order to encompass this variability in our scenarios, we utilise Gaussian (normal) distributions with different values of mean and variance. Selecting a normal distribution to describe the thresholds of the population
TABLE II

TEST RESULTS FOR THE 2 GW LOAD LOSS SCENARIO WITH SEVERAL THRESHOLDS, VALUES OF VARIANCE, PENETRATION OF PV. THE NETWORK LOAD SCENARIO WAS $220 \mathrm{GW}$

\begin{tabular}{|c|c|c|c|c|}
\hline$\%$ PV & Mean under & Mean over & Variance & Load Shedding \\
\hline $10 \div 40$ & 47.5 & 51.5 & $0.1 \div 0.5$ & no \\
\hline 10,20 & 49.5 & 50.5 & $0.05 \div 0.25$ & no \\
\hline 40 & 49.5 & 50.5 & $0.05 \div 0.25$ & var. 0.25 \\
\hline$\sim 10 \%$ & $49.8 \div 49.0$ & 50.2 & $1 \div 9$ & var. 1 \\
\hline
\end{tabular}

TABLE III

TEST RESULTS FOR THE 3 GW POWER LOSS SCENARIO WITH SEVERAL THRESHOLDS, VALUES OF VARIANCE, PENETRATION OF PV. THE NETWORK LOAD SCENARIO WAS $220 \mathrm{GW}$

\begin{tabular}{|c|c|c|c|c|}
\hline$\% \mathrm{PV}$ & Mean under & Mean over & Variance & Load Shedding \\
\hline $10 \div 40$ & 47.5 & 51.5 & $0.1 \div 0.5$ & no \\
\hline 10,20 & 49.5 & 50.5 & $0.05 \div 0.25$ & no \\
\hline 40 & 49.5 & 50.5 & $0.05 \div 0.25$ & yes \\
\hline$\sim 10 \%$ & $49.8 \div 49.0$ & 50.2 & $1 \div 9$ & var. $1 \div 6$ \\
\hline
\end{tabular}

allows to account for the following device characteristics: we consider the ageing, the different manufactures of devices, and assume a random deterioration of the performance of the panels; furthermore we can describe a noisy network where each panel samples the frequency imprecisely, under an additive disturbance.

Time delays are modelled so that the minimum amount of time for a panel to turn on is 20 seconds, whereas its maximum is strictly fixed at 40 seconds. Whilst the latter maximum is handled as a deterministic quantity, the 20-second side of the range is described via a geometric distribution, which can be considered as the discrete-time analogue of the continuous-time exponential distribution. In other words, a PV panel has a positive probability of switching to state ON over a time horizon bound by 40 seconds: at each time step of this horizon, the specific probability is extracted from the geometric distribution.

Special attention is given to value of $49.2 \mathrm{~Hz}$, according to requirements in [8]. In particular, if the frequency trips below the limit of $49.2 \mathrm{~Hz}$ an automatic procedure of loadshedding must be activated. Whilst we did not designed any load shedding procedure in our models, we checked when the network frequency tripped below this critical value, and implicitly assumed that dedicated control systems cause the activation of the load shedding procedure.

TABLE IV

TEST RESULTS FOR THE 2 GW LOAD LOSS SCENARIO WITH SEVERAL THRESHOLDS, VALUES OF VARIANCE, PENETRATION OF PV. THE NETWORK LOAD SCENARIO WAS $440 \mathrm{GW}$.

\begin{tabular}{|c|c|c|c|c|}
\hline$\%$ PV & Mean under & Mean over & Variance & Load Shedding \\
\hline $10 \div 40$ & 47.5 & 51.5 & $0.1 \div 0.5$ & no \\
\hline 10,20 & 49.5 & 50.5 & $0.05 \div 0.25$ & no \\
\hline 40 & 49.5 & 50.5 & $0.05 \div 0.25$ & var. 0.25 \\
\hline$\sim 5 \%$ & $49.8 \div 49.0$ & 50.2 & $1 \div 9$ & var. 1,2 \\
\hline
\end{tabular}


TABLE V

TEST RESULTS FOR THE 3 GW POWER LOSS SCENARIO WITH SEVERAL THRESHOLDS, VALUES OF VARIANCE, PENETRATION OF PV. THE NETWORK LOAD SCENARIO WAS $440 \mathrm{GW}$.

\begin{tabular}{|c|c|c|c|c|}
\hline$\%$ PV & Mean under & Mean over & Variance & Load Shedding \\
\hline $10 \div 40$ & 47.5 & 51.5 & $0.1 \div 0.5$ & no \\
\hline 10,20 & 49.5 & 50.5 & $0.05 \div 0.25$ & no \\
\hline 40 & 49.5 & 50.5 & $0.05 \div 0.25$ & yes \\
\hline$\sim 5 \%$ & $49.8 \div 49.0$ & 50.2 & $1 \div 9$ & no \\
\hline
\end{tabular}

We test three thresholds scenarios, ranging from the newly published European regulation to a narrow working frequency interval, and finally a composite scenario of three Gaussian distributions. Tables II, III, IV, V show the results of our tests, as a function of the percentage of solar penetration in the network, and of the mean value and variance of the distributions. When the network frequency trips below 49.2 $\mathrm{Hz}$ we assume the procedure of load shedding is activated and we report it in the Load Shedding column. When this happens in experiments with a specific variance, we report its value. In the following we describe the outcomes of four different practical scenarios of thresholds distribution.

A. European regulation Scenario: We follow the European Community guidelines [6] as the safest possible scenario. According to these guidelines, inverters should work within the interval $[47.5,51.5] \mathrm{Hz}$, so we set these two values as the mean for the under- and over- thresholds distributions, respectively. In order to simulate decreasing noisy measurements of the frequency we set five experiments, with different variances for the distributions, from 0.1 to $0.5 \mathrm{~Hz}^{2}$ with a step of 0.1 for each experiment. Such frequency threshold values ensure a stable network in every tested configuration and for both the power generation loss and load loss incidents. In Figure 4 the frequency response is shown with $40 \%$ of solar penetration and in the case of a $2 \mathrm{GW}$ load loss.

B. Narrow interval Scenario: We set the thresholds around the values of $49.5 \mathrm{~Hz}$ and $50.5 \mathrm{~Hz}$, resulting in a $500 \mathrm{mHz}$ band around the nominal frequency. Five scenarios for the variance are investigated, with values ranging from 0.1 to 0.25 $\mathrm{Hz}^{2}$ with a step of 0.05 (step values are smaller compared to the previous scenario, in view of the proximity of the average with the nominal frequency). These experiments show that only in the case of a $40 \%$ of solar production there is a risk of load shedding, both in the power loss and load loss incident. Figure 5 shows the frequency drop in case of the $3 \mathrm{GW}$ power generation failure. Cases where solar production is set to $10 \%$ or $20 \%$ do not lead to load shedding events.

C. A more realistic Scenario: In order to achieve a more faithful description of the heterogeneity of the grid, we slightly modify the settings used in the previous two scenarios. In this case we employ data from European countries [5] as a starting point. We assume to have a given amount of panels that are activated according to the regulation of the installation year, as shown in Table VI. We assume also that the condition to comply with the regulations is to have a threshold equal to
TABLE VI

PARTITION OF UNDERFREQUENCY THRESHOLDS AND ASSOCIATED LOAD IN MegaWatTs.

\begin{tabular}{|c|c|c|c|c|c|}
\hline Underfrequency threshold & 49.8 & 49.7 & 49.5 & 49.0 & 47.5 \\
\hline Overfrequency threshold & 50.2 & 50.2 & 50.2 & 50.2 & 50.2 \\
\hline MW & 1000 & 2000 & 4500 & 4000 & 8500 \\
\hline
\end{tabular}

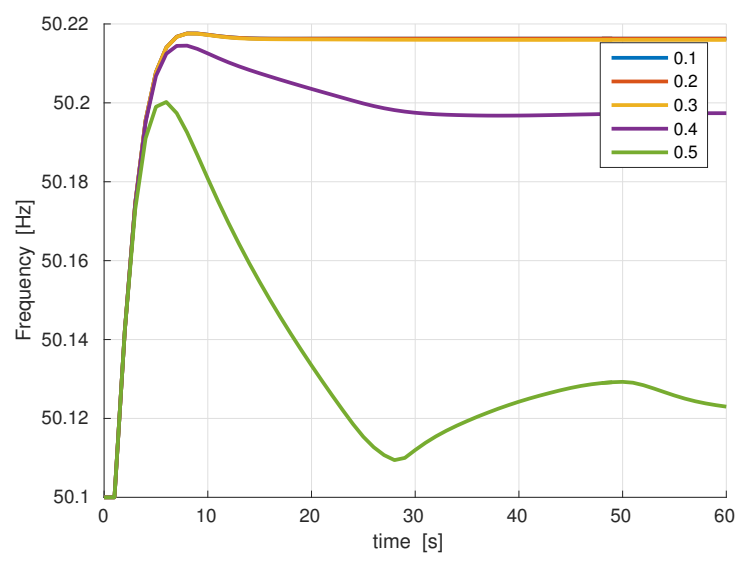

Figure 4. Simulations for a load loss of $2 \mathrm{GW}$ with several values of variance of the Gaussian distribution for the thresholds. The mean value for the distributions are $47.5 \mathrm{~Hz}$ and $51.5 \mathrm{~Hz}$ in underfrequency and overfrequency, respectively, with a $40 \%$ penetration of solar power generation.

the limit or further away from the nominal frequency. As an example, panels installed with the $49.5 \mathrm{~Hz}$ underfrequency limit can have a threshold $f_{u f} \leq 49.5$. Similarly, panels are allowed to have $f_{o f} \geq 50.2$ in the $50.2 \mathrm{~Hz}$ overfrequency case. In order to probabilistically describe a similar situation we utilise a $\chi^{2}$ distribution. In contrast with the previous scenarios, the $\chi^{2}$ distribution is not symmetric around its average value. We set a limit value using values of Table VI and distribute the corresponding amount of MW according to the probability distribution. The total amount of MW is close to $10 \%$ of solar penetration in the $220 \mathrm{GW}$ load demand, $5 \%$ in the $440 \mathrm{GW}$. We test five values of variance, ranging from 1 to $9 \mathrm{~Hz}^{2}$, with a step of 1 . Note that increasing variance leads to distributing the population thresholds away from the nominal frequency. Simulations show that increasing the variance enhances the reliability of the grid, as depicted in Figure 6 for the $3 \mathrm{GW}$ generation loss. Small values of variance cause the network to trip below the $49.2 \mathrm{~Hz}$ limit, leading to a load shedding; higher values, that is higher heterogeneity, keep the electric grid away from failure.

\section{Discussion AND CONCLUSIONS}

In this paper we discussed two Markov models for the frequency behaviour of a large heterogeneous population of photovoltaic panels. We pair these models with a discrete-time equation representing the frequency dynamics of the electric grid: this allows to consider the feedback interconnection between the panels and the grid. Having established this modelling framework, in this work we focus on testing the stability of a network representing the EU Continental grid. In two 


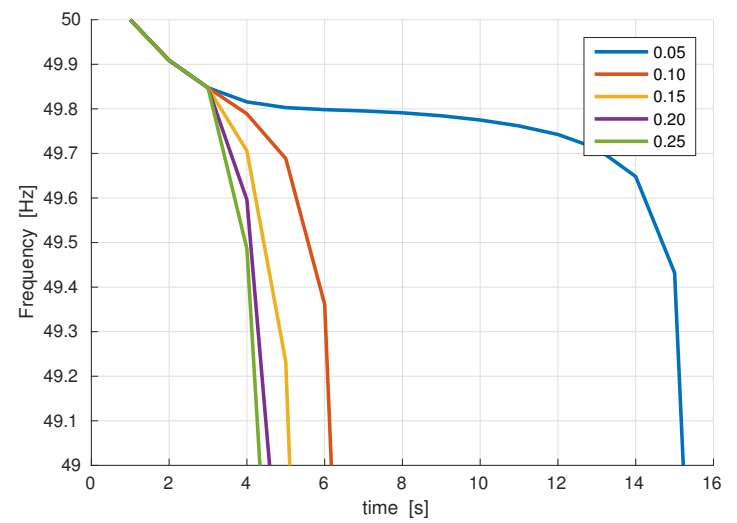

Figure 5. Simulations for a power generation loss of $3 \mathrm{GW}$ with several values of variance of the Gaussian distribution for the thresholds. The mean value for the distributions are $49.5 \mathrm{~Hz}$ and $50.5 \mathrm{~Hz}$ in underfrequency and overfrequency, respectively, with a $40 \%$ penetration of solar power generation.

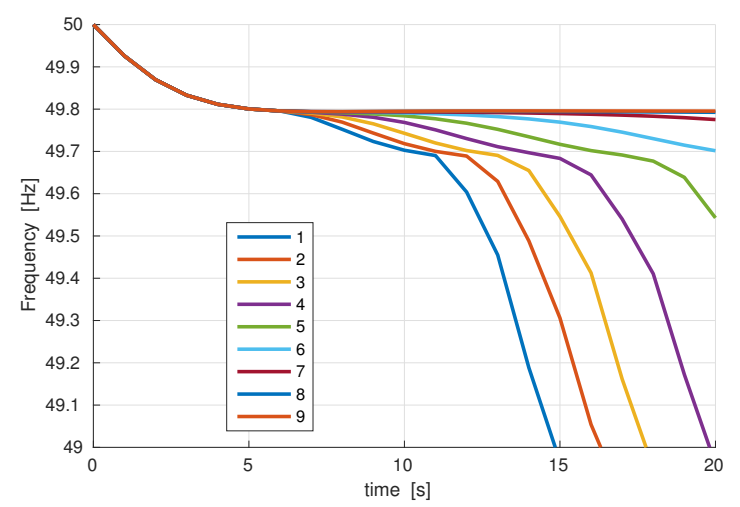

Figure 6. Simulations for a power generation loss of $3 \mathrm{GW}$ with several values of variance of the $\chi^{2}$ distribution for the thresholds. The solar power penetration is around $10 \%$ in a $220 \mathrm{GW}$ load demand network.

scenarios of generation loss and load loss, we run experiments with different distributions of population thresholds.

Experiments show a dependence between load shedding and the variance of the chosen distributions. A Gaussian distribution has a symmetric shape around its average value. When its variance increases, the tails on both sides spread out. If frequency thresholds are distributed according to a Gaussian, increasing the variance of the thresholds distribution cause a higher number of panels (represented by the tails of the distribution) to have thresholds further from the average value. As a consequence, we observe more panels with a narrow working interval around the nominal frequency. As more panels have a narrow working interval, the network is more likely to fail, i.e. the frequency to trip below $49.2 \mathrm{~Hz}$. This issue will increase with the number of photovoltaic devices installed, unless new panels have a larger working interval. Note that we have assumed a noiseless frequency signal, which is not the case in practice. Probabilistic thresholds can be thought as a description of a device with a constant threshold plus a noisy sensor. In this sense, the Gaussian distribution is the most appropriate choice. The $\chi^{2}$ are more useful to set a minimum target of performance (minimum working interval) leaving room for noise at values of frequency further from $f_{0}$. In case of the $\chi^{2}$ distribution, increasing variance corresponds to larger thresholds. Experiments show that increased heterogeneity guarantees a reliable network, while a significant portion of panels with a fixed threshold value complying old regulations might jeopardize the regular operation of the network. In this sense, if countries follow the new European regulations, the risk of frequency tripping below a critical value is dramatically reduced, regardless of whether a power generation or a load loss incident happen.

We conclude by advocating the need for heterogeneous thresholds, towards having larger working intervals, in order to guarantee the stability of the electric network in case of normal incidents. Household solar devices have already been quite widespread in the market: this population is rarely participant in the retrofit programs proposed by the European Community, and we imagine them less frequently serviced than industrial installations. The ageing process will bring less reliable devices that generate noisy frequency samples which influence the population behaviour. It is then vital to install new devices compliant with the new European regulations.

Future development in the modelling of population of solar panels should consider PV devices with different power output, encompassing the network topology, local weather conditions, and their different sizes. Furthermore, panels should not only react to the frequency, but also be able to interact with it actively. Existing approaches from the wind power area, such as synthetic inertia, may be installed in the photovoltaics via a distributed control architecture.

\section{REFERENCES}

[1] ENTSO-E, "Statistical factsheet," Tech. Rep., 2016.

[2] H. Wirth, "Recent facts about photovoltaics in Germany," Fraunhofer ISE, Tech. Rep., 2016.

[3] Solar Power Europe, "Global market outlook for solar power 20162020,” Tech. Rep., 2016.

[4] "Legal comparison of PV regulations," http://www.reslegal.eu/comparison-tool/, accessed: 04/04/2017.

[5] ENTSO-E, "Dispersed generation impact on CE region, dynamic study," Tech. Rep., 2014.

[6] E. Commission, "Commission regulation (EU) 2016/631 of 14th april 2016," Tech. Rep., 2016.

[7] A. Peruffo, E. Guiu, P. Panciatici, and A. Abate, "Aggregated markov models of a heterogeneous population of photovoltaic panels," International Conference on Quantitative Evaluation of Systems, pp. 72-87, 2017.

[8] ENTSO-E, "Policy 1: load-frequency control and performance," Tech. Rep., 2009.

[9] H. Tiam, F. Mancilla-David, and K. Ellis, "A detailed performance model for photovoltaic systems," NREL, Tech. Rep., 2012.

[10] J. G. Kassakian, R. Schmalensee, G. Desgroseilliers, T. D. Heidel, K. Afridi, A. Farid, J. Grochow, W. Hogan, H. Jacoby, J. Kirtley et al., "The future of the electric grid," Massachusetts Institute of Technology, Tech. Rep, pp. 197-234, 2011.

[11] E. B. Iversen, J. M. Morales, and J. K. Moller, "Probabilistic forecasts of solar irradiance by stochastic differential equations," Envirometrics, vol. 25, Apr. 2014.

[12] M. Jung, O. Wiss, and B. Lazpita, "Analyses et conclusions - Tests en sur-frequence," RTE, Tech. Rep., 2016.

[13] — "Analyses et conclusions - Tests en sous-frequence," RTE, Tech. Rep., 2016.

[14] ENTSO-E, "Continental europe operation handbook - glossary," Tech. Rep., 2004.

[15] ㄴ. "Network code on operational security," Tech. Rep., 2013. 